\title{
AOA and TDOA-Based a Novel Three Dimensional Location Algorithm in Wireless Sensor Network
}

\author{
Tian Zhanwei*, Hao Huicheng and Shi Yan \\ Northeast Agricultural University, Haerbin, Heilongjiang 150030, China
}

\begin{abstract}
Combining angle measurement algorithm based on wave interference in two-dimensional space with positioning algorithm based on time difference of arrival, this study proposes a Wireless Sensor Networks localization algorithm for three-dimensional space. First, the algorithm measures the angle and distance of beacon nodes and test nodes, and then calculates the precise coordinates of the test node. The algorithm complexity is low, just needing at least three beacon nodes. As it uses distributed computing, so it has high positioning accuracy. Simulation results show that the algorithm can accurately calculate the position of the sensor nodes in three-dimensional space.
\end{abstract}

Keywords: Angle measurement, three-dimensional location, time difference of arrival, WSN.

\section{INTRODUCTION}

Wireless Sensor Network (WSN) is a technology combining with microelectronics, communications, computer and sensor technology. It has a wide range of applications in civil, military, industrial and other commercial areas. As one of the key technologies of WSN, positioning technology not only plays an important role in the basic application of WSNs, but also provides the basis for monitoring and tracking of targets.

Most existing localization algorithms have positioning for flat space. WSN localization methods can be divided into range-based localization algorithm and distance-independent localization algorithm [1-3]. Range-based localization algorithm needs to measure node-point distance or angle information between the nodes [4]. Commonly used ranging techniques include TOA (Time of Arrival) [4], TDOA (Time Different on Arrival) [5], RS-SI (Received Signal Strength Indicator), AOA (Angle of Arrival) [6] and so on. Hardware configuration requirements of such methods of node are higher than the Range-Free method, but their positioning accuracy is better than Range-Free algorithm. Range-Free positioning does not required distance and angle information, and can only achieve targeted based network connectivity and other information. Typical algorithms include DV-hop, APITE etc. Although such algorithms have low hardware cost and low power consumption advantages, yet their positioning accuracy is low.

Practical applications of WSN nodes are often distributed in three-dimensional space. Research on the threedimensional spatial orientation will be more in line with the actual application of the nodes. Distributed low computational complexity three-dimensional positioning methods are more practical and have more development potential than $2 \mathrm{D}$ methods.

\footnotetext{
*Address correspondence to this author at the Northeast Agricultural University, Haerbin, Heilongjiang150030, China; Tel: 18986139113 ; E-mail: hunter2011@foxmail.com
}

This paper presents a three-dimensional joint WSNs localization algorithm based on wave interference sensor angle measurement (AOA) and time difference of arrival (TDoA). The algorithm has a high precision; therefore it can effectively estimate three-dimensional space position sensor nodes. The algorithm is divided into three steps: firstly, using the measurement algorithm based on wave interference angle sensor to calculate the angle of beacon nodes and unknown nodes; then using the measured angle and distance, using the proposed derived formula, to calculate the precise coordinates of the unknown node.

\section{THE BASIC CONCEPT OF WSN NODE LOCALIZATION}

In sensor network, each node determines its own position in the space of a space coordinate system called node localization process [7-10]. Due to limited energy WSNs, large number, low cost, each sensor node is equipped with a GPS receiver, or prior to the specified location information for each node, that are unrealistic. Therefore, we can only make a small part of the node assembly positioning device, or prior to the node at the specified location coordinates. These a few nodes that through some means strive to know their location are called beacon nodes or anchor nodes. Due to restrictions of cost and energy consumption, the anchor nodes in the network constitute a small proportion. In the sensor network, in addition to the known location information of beacon nodes, the nodes that need some kind of algorithm to calculate the location information, are called unknown nodes.

Beacon nodes are a small proportion in a network node. It can get its exact location by means of portable GPS positioning equipment, since a reference node location is unknown. To determine the location of unknown nodes, beacon node broadcasts its own location information signal to the unknown node.

WSN node localization problem can be expressed as: relaying on the limited position known beacon node to determine the location of other unknown nodes in layout 
area, and building a certain spatial relationship between the sensor nodes.

For different wireless location systems, methods and techniques to achieve a location are different. From the principle of speaking, wireless positioning system generally consists of the following three steps:

In the first step, measure one or several parameters (amplitude, frequency, phase, propagation time) of radio signals. According to the radio wave propagation characteristics, the electrical parameter measurement is converted to a distance, the distance difference and angle of arrival, etc. used to represent the positional relationship.

In the second step, use a variety of algorithms or techniques to achieve the position estimate.

In the third step, the optimization of the estimated value is done.

The same applies for WSNs localization of these three steps.

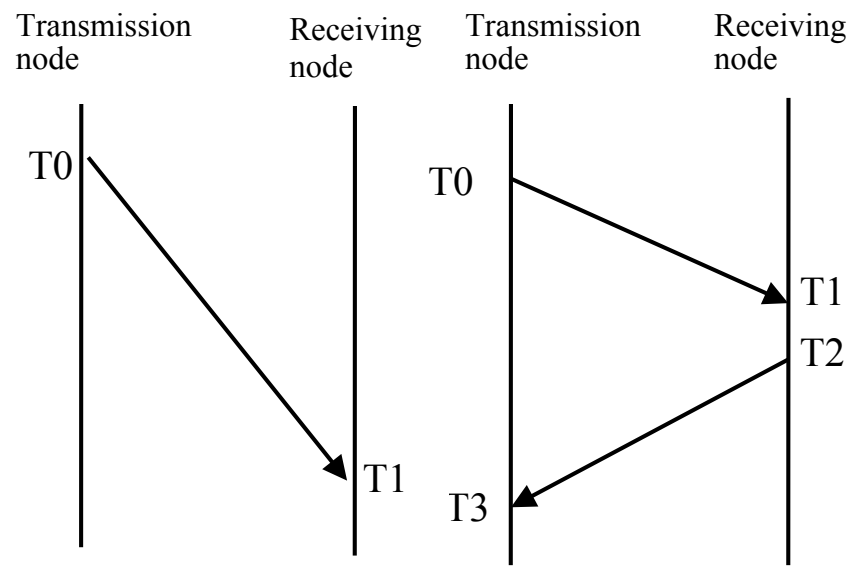

Fig. (1). Sketch figure of TOA location.

\section{ANGLE AND DISTANCE MEASUREMENT METHOD OF SENSOR NODES IN THREE- DIMENSIONAL SPACE}

\subsection{Angle Measurement Method of Sensor Nodes}

In three-dimensional space, the angle of measured node to beacon node is the angle between the line $P A$ and the plane $X O Y$. As shown in Fig. (1), passing the point $P$ do $P P A \perp$ plane $X O Y$, intersects with the plane passing point $A$ and paralleling to the plane $X O Y$ at points $P A$. Connect $P P A$, then $\angle P A A P$ is the angle from node $P$ to node $A$.

We use the sensor angle measurement method based on interference wave in two-dimensional space to perform the sensor angle measurement in three-dimensional space (Fig. 2).

As shown in Fig. (3), there are two ultrasonic transmitters ( $S 1$ and $S 2$ ) having the distances of $2 r$ and $S 1 S 2 \| Z$ on the beacon nodes. They emit the same frequency, in the same initial phase ultrasound. The two ultrasonic transmitters will experience interference at the point $P$. Passing point $P$ do $P P_{A}$ plane $X O Y$, connects $O P_{A}$. It can be proved by the three-dimensional geometry knowledge: $O P_{A}$ is the vertical line of transmitters $S 1$ and $S 2$, and $S 1, S 2, P$ and $P_{A}$ are at the same plane.

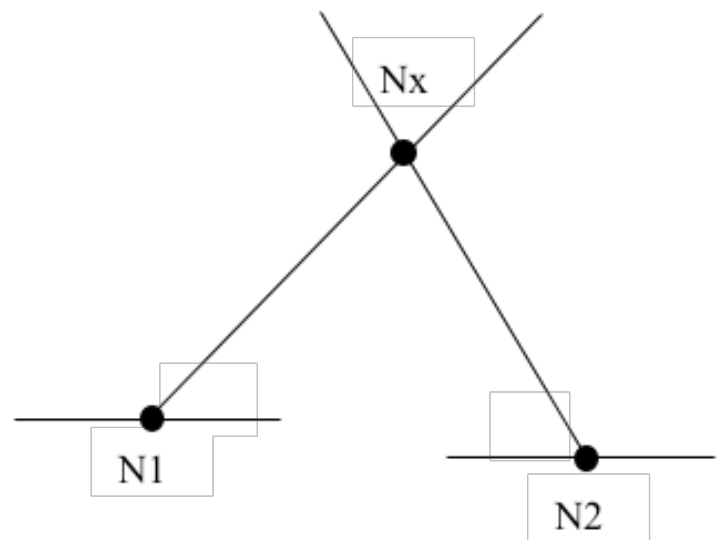

Fig. (2). Angle diagram of three-dimensional space between nodes.

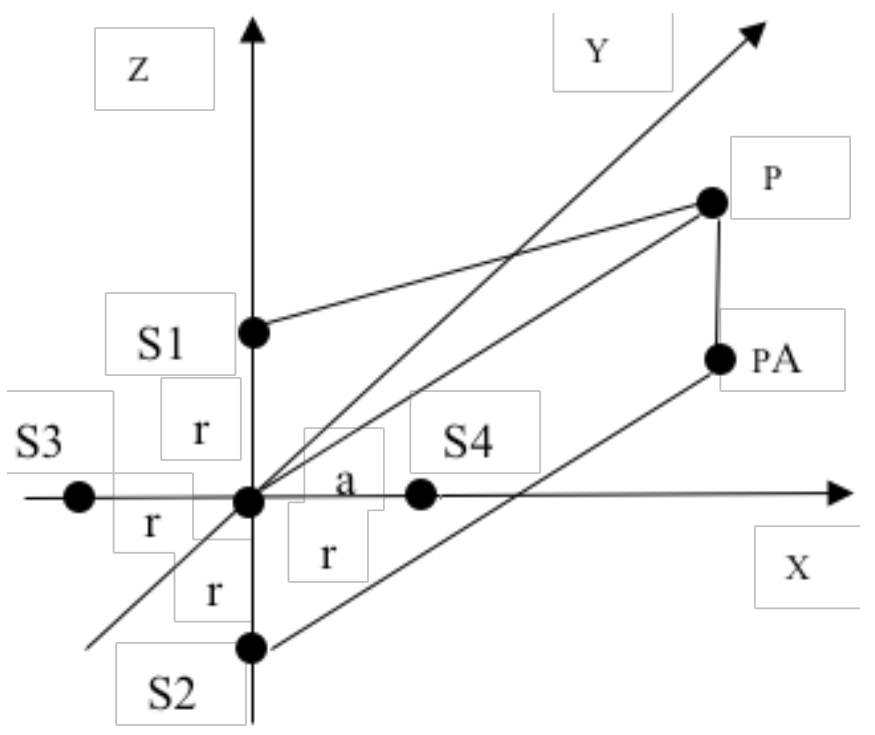

Fig. (3). Schematic positions of beacon nodes and unknown nodes.

So, we can use the formula of document [2] in plane $S 1 S 2 P P_{A}$ :

$\sin (\alpha)=\frac{v}{2 r \cdot T \cdot f^{\prime}}=\frac{v}{2 r \cdot f^{\prime}} \mathrm{F}$

Of which, $v$ is the propagation velocity of sound waves in the air; $2 r$ is the distance between two ultrasonic transmitters; $f$ is the rate of change of frequency of the ultrasonic signal source; $T$ is the synthesized wave intensity change cycle of point $P$ when source frequency rate changes with rate $f^{\prime} ; \mathrm{F}$ is the change frequencies of corresponding point $P$. As long as measuring variation period or frequency of the composite wave intensity, since the other parameters are known, it can calculate the angle $\alpha$ at the point $P$ with respect to beacon node. 


\subsection{Distance Measurement Method of Sensor Nodes}

The method of calculating the distance between two nodes in the positioning mechanism based on TDOA in three-dimensional space, is equally applicable to AOA in three-dimensional space. Transmitting nodes simultaneously transmit radio frequency signals and ultrasonic signals. The receiving node records the arrival time, $\mathrm{T}_{1}$ and $\mathrm{T}_{2}$ of two signals. Known radio-frequency signals and ultrasonic propagation velocity are $c_{1}$ and $c_{2}$. So the distance between

two points is $1=\left(\mathrm{T}_{2}-\mathrm{T}_{1}\right) \cdot \frac{c_{1} c_{2}}{c_{1}-c_{2}}$.

The algorithm presents an issue of dead space that cannot be measured, where there is a threshold time $\mathrm{T}_{0}$ of Interference wave cycle of point $P$. For the angle $\theta<\arcsin \left(\frac{v}{2 r \cdot T_{0} \bullet f^{\prime}}\right)=\theta_{0}$, due to its interference wave intensity, was less than one cycle, it can't be measured.

Original algorithm placed one pair of ultrasonic transmitters $S 1$ and $S 2$ on beacon nodes. Now, we add a pair of ultrasonic transmitters $S 3$ and $S 4$. Both the transmitters' performance parameters are the same, and positions perpendicular to each other. The angles between them and the point $P$ are $\theta_{1}$ and $\theta_{2}$, as shown in Fig. (3).

Because $\theta_{1}$ and $\theta_{2}$ are mutually complementary angle, as long as the angle $\theta_{0}$ is less than $45^{\circ}$, on at least one of $\theta_{1}$ and $\theta_{2}$ can be calculated.

For achieving $\theta_{0}<45^{\circ}$, according to the formulas (2-17) deduced: $\Delta f>\frac{\sqrt{2}}{2} \cdot \frac{v}{r}$.

$v$ is the propagation velocity (approximately equal to $340 \mathrm{~m} / \mathrm{s}$ ) of ultrasonic wave in the air; $2 r$ is the distance between two ultrasonic transmitters (By node hardware restrictions, general $0<r<0.1 m) ; f^{\prime}$ is the difference between the cut-off frequency and the initial frequency of the ultrasonic signal (Ultrasonic frequency range is from $2 \times 10^{4}$ to $2 \times 10^{8} \mathrm{~Hz}$ ). Fig. (4) is the graph of function $\Delta f>\frac{\sqrt{2}}{2} \cdot \frac{v}{r}$ in the interval $r \in(0,0.1)$. In the figure, the values of the curve at the top is the values of $f$ and $r$ In line with the formula (2).

\section{THREE-DIMENSIONAL POSITIONING ALGORI- THM PRINCIPLE OF WSN}

As shown in Fig. (4), the known coordinates of three beacon nodes $\mathrm{A}, \mathrm{B}$ and $\mathrm{C}$ are respectively $\left(\mathrm{x}_{a}, \mathrm{y}_{a}, \mathrm{z}_{a}\right)$, $\left(\mathrm{x}_{b}, \mathrm{y}_{b}, \mathrm{z}_{b}\right)$ and $\left(\mathrm{x}_{c}, \mathrm{y}_{c}, \mathrm{z}_{c}\right)$. The angles of measured node $\mathrm{P}$ relative to the nodes $\mathrm{A}, \mathrm{B}$ and $\mathrm{C}$ are respectively $\alpha, \beta$ and $\gamma$. According to the measurement algorithm based on sensor angle wave interference, the distances of node $\mathrm{P}$ to $\mathrm{A}, \mathrm{B}$ and
$\mathrm{C}$ are respectively $P_{A}, P_{B}$ and $P_{C}$ can be measured. Based on the TDOA based positioning mechanism, the distances measured are respectively $l_{a}, l_{b}$ and $l_{c}$. Assuming the coordinate of point $\mathrm{P}$ is $(\mathrm{x}, \mathrm{y}, \mathrm{z})$.

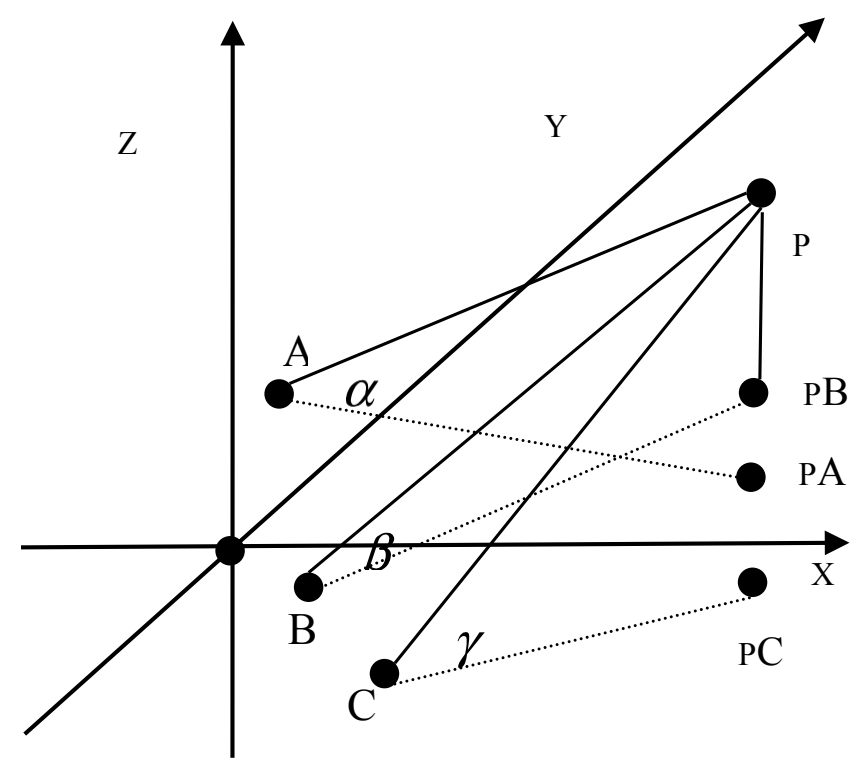

Fig. (4). Schematic positions of beacon nodes and unknown nodes in three dimensional space.

Because $\triangle P A P_{A}$ is a Right triangle, so the formula is:

$$
\begin{aligned}
& \left(\mathrm{x}-\mathrm{x}_{a}\right)^{2}+\left(\mathrm{y}-y_{a}\right)^{2}+\left(\mathrm{z}-z_{a}\right)^{2}=\left(l_{a}\right)^{2} \\
& \left(\mathrm{x}-\mathrm{x}_{b}\right)^{2}+\left(\mathrm{y}-y_{b}\right)^{2}+\left(\mathrm{z}-z_{b}\right)^{2}=\left(l_{b}\right)^{2} \\
& \left(\mathrm{x}-\mathrm{x}_{c}\right)^{2}+\left(\mathrm{y}-y_{c}\right)^{2}+\left(\mathrm{z}-z_{c}\right)^{2}=\left(l_{c}\right)^{2} \\
& \left(\frac{z-z_{a}}{l_{a}}\right)^{2}=(\sin \alpha)^{2} \\
& \left(\frac{z-z_{b}}{l_{b}}\right)^{2}=(\sin \beta)^{2} \\
& \left(\frac{z-z_{c}}{l_{c}}\right)^{2}=(\sin \gamma)^{2}
\end{aligned}
$$

By equation (4), (6), (8), we can obtain 6 solutions of $z$. The average of the three reasonable solutions is the $z$ value. In this article, all the sensor nodes are placed in the first quadrant of the coordinate space, so $\mathrm{z}$ value must be positive, negative solution is void. Because there is an error between the measured angle and distance of the node, therefore by equations (4), (6), (8) the obtained solutions of $\mathrm{z}$ are not equal, but will be very close. So, taking the average of the closest 3 value of 6 solutions is the value of the solution.

By putting equations (4), (6), (8) into the formulas (3), (5), (7), a new set of equation (9) can be obtained, which is the WSN node localization trilateration measurement method.

$$
\begin{aligned}
& \left(\mathrm{x}-\mathrm{x}_{a}\right)^{2}+\left(\mathrm{y}-\mathrm{y}_{a}\right)^{2}=\left(l_{a} \cdot \cos \alpha\right)^{2} \\
& \left(\mathrm{x}-\mathrm{x}_{b}\right)^{2}+\left(\mathrm{y}-y_{b}\right)^{2}=\left(l_{b} \cdot \cos \beta\right)^{2}
\end{aligned}
$$




$$
\left(\mathrm{x}-\mathrm{x}_{c}\right)^{2}+\left(\mathrm{y}-y_{c}\right)^{2}=\left(l_{c} \cdot \cos \gamma\right)^{2}
$$

This algorithm uses only three beacon nodes, so it is possible to accurately measure the coordinates of the unknown node. However, due to limitations of the ultrasonic signal transmission distance, a number of beacon nodes may be distributed within a target area. When a node receives information of an unknown angle sensor node near the $\mathrm{N}$ $(\mathrm{N}>3$ ) beacon, this information is transmitted between nodes and distance, the node can choose to receive information of the nearest three beacon nodes, giving up the rest of the beacon nodes by sending a message. Because ultrasonic signal form larger environmental impact, the closer the subject of outside interference, the smaller, more accurate data to calculate the coordinates of the more accurate self.

\section{THE MEASUREMENT RESULTS}

\subsection{Setting the Simulation Environment}

Simulation environment is set as follows: experimental environment under normal temperature conditions; Wireless signal propagation speed is $c_{1}=3 \times 10^{8} \mathrm{~m} / \mathrm{s}$; Ultrasonic wave propagation velocity is $c_{2}=340 \mathrm{~m} / \mathrm{s}$; The amplitude of the ultrasonic signal source is set to 1 , the initial phase is zero; Start frequency is $f_{0}=2 \times 10^{4} \mathrm{HZ}$; The stop frequency is $f_{t}=2 \times 10^{5} \mathrm{HZ}$; The time interval is $t_{0}=1 \mathrm{~s}$. So there is

$f^{\prime}=\frac{f_{t}-f_{0}}{t_{0}}=18 \times 10^{4} \mathrm{HZ} / \mathrm{s}$

Signal to Noise Ratio is $S N R=15 d B$. Value of $R$ is measured in the range $0 \sim 1.0 \mathrm{~m}$.

On the spatial distribution, set three beacon nodes A, B and $C$, and their coordinates are $(0,0,0),(200,300,0)$ and $(250,250,50)$. Because ultrasonic signal is impacted by the environment, the closer the subject of outside interference, the smaller, more accurate data to calculate the coordinates of the more accurate self. So we set node communication distance as $500 \mathrm{~m}$. In the space of $400 \mathrm{~m} \times 400 \mathrm{~m} \times 50 \mathrm{~m}$ three-dimensional space coordinates in the first quadrant, there are 100 tested randomly distributed nodes.

\subsection{Simulation Results and Performance Analysis}

By measuring a test node, simple description of the entire simulation process is given.

Randomly assign a test point $P$, whose real coordinate is $(280,150,35)$. Simulate the case of $0-0.1 \mathrm{~m}$. Intensity variation waveform of the composite wave of ultrasonic signals emitted by the beacon nodes $A$ at point $P$ is as shown in Fig. (5).

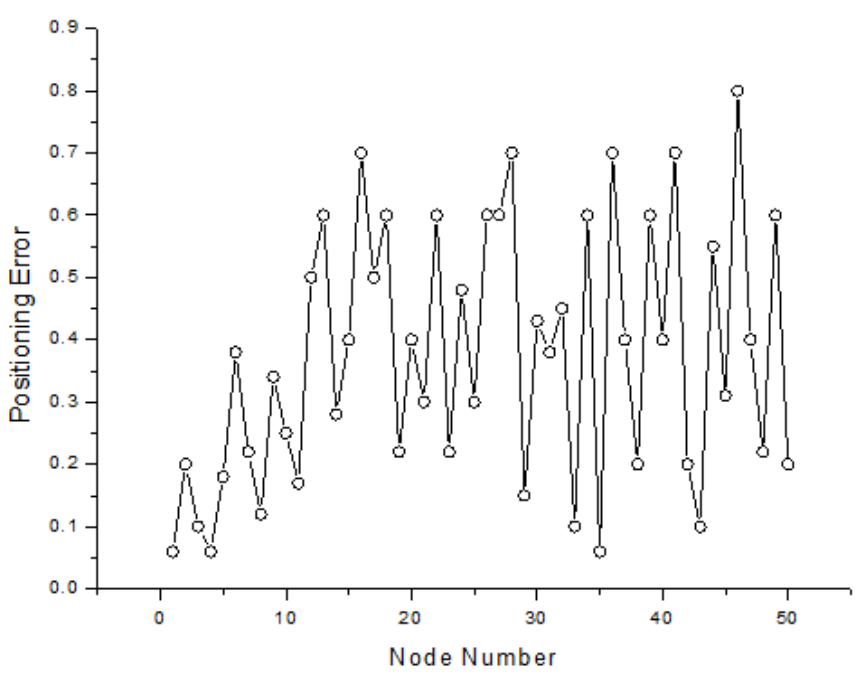

Fig. (5). Ultrasonic of point A intensity wave.

Do FFT transform to the intensity of the composite wave. Set the sampling frequency $f s=2^{11}$, Sampling point is $N=2^{12}$. From the data FFT, change can be measured, the frequency of the composite wave intensity change is $F=422 \mathrm{HZ}$, taking into formula to obtained $\theta_{a}=0.0884$. In the same way, calculate the angles of point $P$ to beacon nodes $\mathrm{B}$ and $\mathrm{C}$ that are $\theta_{b}=198.6454$ and $l_{c}=129.4603$. Finally, combine with the angle and distance measurements to calculate the coordinates of the point that is $P^{\prime}=(307.9403,136.0693,30.0330)$. Distance measured point coordinates and real coordinates as measurement error. Error is $\eta=\left|P P^{\prime}\right|=0.0973 m$. Positioning accuracy using realworld coordinates and the coordinates of the measured distance to the percentage deviation distance through a hearing to represent, equal that $\eta / 500=0.0195 \%$. Clearly, the accuracy is relatively high.

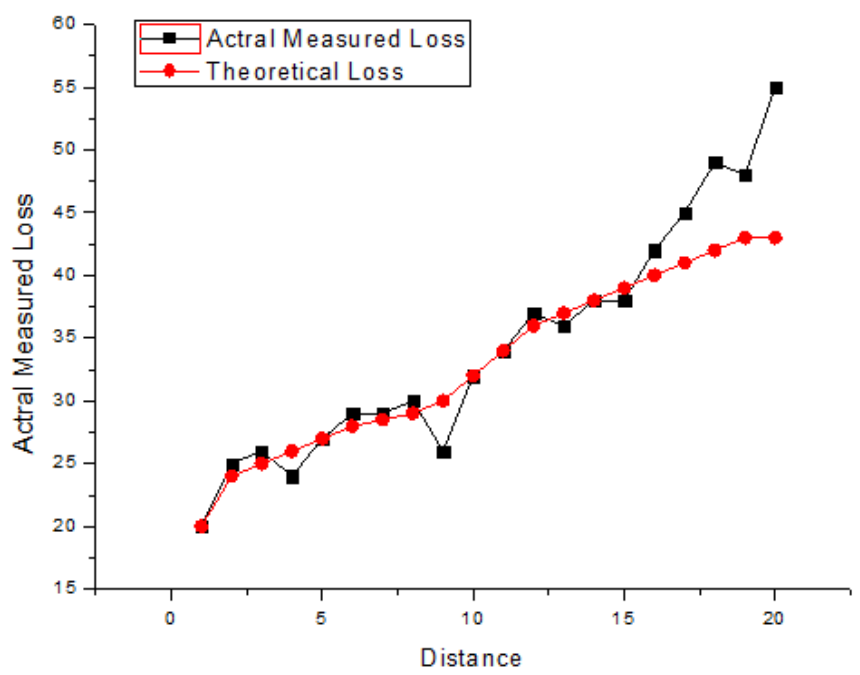

Fig. (6). The relationship between signal attenuation and distance.

Experiments (Fig. 6) show that, the average signal attenuation in the line of sight distance, that is the average value of received signal strength and emission power 
difference, is close to the range of about 10 meters, the experiment results accord well with the theoretical attenuation model. But with the increase of distance, change of signal attenuation deviated from the empirical model. That is, in the real environment, the short distance, according to the received signal strength to measure the emission power, is more reliable in empirical model.

In fact, during the experiment it can be found that the received signal strength has a very distinct time-varying characteristic. Corresponding to different distances, the change of its location error is a random process. Fig. (7) shows that, corresponding to different distances, the line of sight distance of the normal number of errors is estimated from the empirical model and the actual distance, i.e. ranging error. As can be seen from the figure, when the distance increases to a certain extent, the ranging error also increases significantly. And in the short distance (about $10 \mathrm{~m}$ in the present experiment), the vast majority of the errors is maintained at about $10 \%$. Description in the case of sight distance of close range accuracy is more reliable.

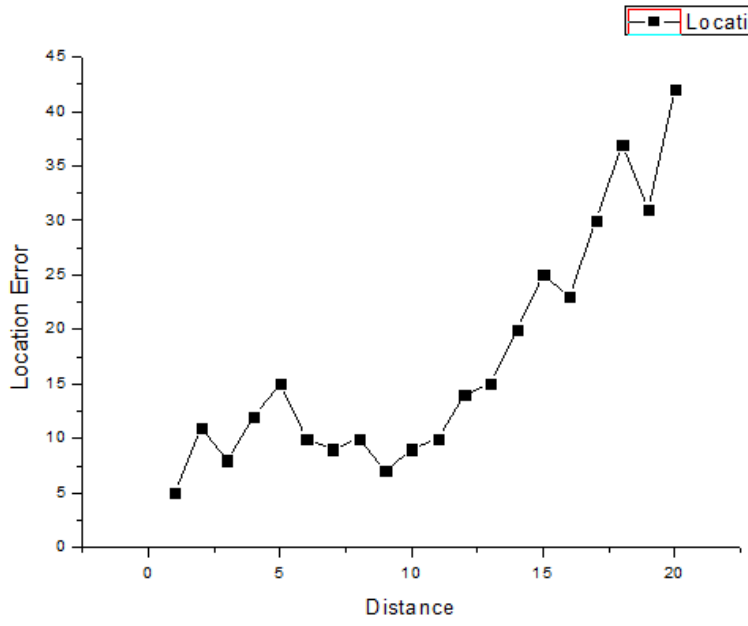

Fig. (7). The relationship between signal attenuation and distance.

\section{CONCLUSION}

Simulation results show that the proposed combined localization algorithm for WSNs based on wave interference angle measurement (AOA) and time difference of arrival (TDOA) in three-dimensional space, has a lower complexity. It just needs at least three beacon nodes. It has a high precision arithmetic, so it can effectively estimate the location of the sensor nodes in three-dimensional space. Meanwhile, this method has the disadvantage of locating algorithm based on wave interference angle measurement and TDOA, that's ultrasonic transmitter may be affected by environmental, temperature and other external factors.

Practical applications of WSN nodes are often distributed in three-dimensional space. Research on the three-dimensional spatial orientation will be more in line with the actual application of the nodes. Distributed low computational complexity three-dimensional positioning methods are more practical and have development potential than 2D methods.

This paper presents a three-dimensional joint WSNs localization algorithm based on wave interference sensor angle measurement (AOA) and time difference of arrival (TDOA). The algorithm has a high precision, and it can effectively estimate three-dimensional space position sensor nodes. The algorithm is divided into three steps: firstly, using the measurement algorithm based on wave interference angle sensor to calculate the angle between beacon nodes and unknown nodes; then using the measured angle and distance, using the proposed derived formula, to calculate the precise coordinates of the unknown node.

\section{CONFLICT OF INTEREST}

The authors confirm that this article content has no conflict of interest.

\section{ACKNOWLEDGEMENTS}

This work is supported by Heilongjiang Province Social Science Fund "Based on the analysis of the social media network information propagation rules, pattern, and mechanism and supervision strategy research" (:13C010).

\section{REFERENCES}

[1] R. Wade, W. Mitehel, and F. Petter, "Ten emerging technologies that will change the world," Technology Review, vol. 106, pp. 22-49, 2003.

[2] J.A. Byme, "21 ideas for $21^{\text {st }}$ Century", Business Week, pp. 78-167, 1999

[3] J. Agre and L. Clare, "An integrated architecture for cooperative sensing networks," Computer, vol. 33, pp. 106-108, 2000.

[4] V. Mhatre and C. Rosenberg, "Design guidelines for WSN s: communication, clustering and aggregation," Ad Hoc Networks, vol. 2, pp. 45-63, 2004.

[5] D. Ganesan, A. Cerpa, W. Ye, Y. Yu, J. Zhao, and D. Estrin, "Networking issues in WSN s," Journal of Parallel and Distributed Computing, vol. 64, pp. 799-814, 2004.

[6] Y. Shang, W. Ruml, Y. Zhang, and M. P. Fromherz, "Localization from mere connectivity," In: Proceedings of the $4^{\text {th }}$ ACM International Symposium on Mobile ad Hoc Networking \& Computing, 2003, pp. 201-212.

[7] T. Hui, W. Shuang, and X. Huaiyao, "Localization using cooperative AOA approach. proceeding of wireless communications", Networking and Mobile Computing, pp. 2416-2419, 2007.

[8] J. Xu, M. Ma, and C. L. Law, "AOA cooperative position localization," In: Global Telecommunications Conference, 2008. IEEE GLOBECOM 2008. IEEE, 2008, pp. 1-5.

[9] S. CaPkun, M. Hamdi, and J.-P. Hubaux, "GPS-Free positioning immobile ad-hoc networks", Cluster Computing, pp.157-167, 2012.

[10] Y. Shang, W. Ruml, Y. Zhang, and M. P. Fromherz, "Localization from mere connectivity," In: Proceedings of the $4^{\text {th }}$ ACM International Symposium on Mobile ad Hoc Networking \& Computing, 2003, pp. 201-212. 